\title{
Protective Effect of Arabinoxylan against Scopolamine-Induced Learning and Memory Impairment
}

\author{
Chang-Yul Kim¹, Gil-Yong Lee ${ }^{1}$, Gyu Hwan Park², Jongwon Lee ${ }^{3, *}$ and Jung-Hee Jang ${ }^{4, *}$ \\ ${ }^{1}$ Department of Pathology, College of Oriental Medicine, Daegu Haany University, Daegu 706-828, \\ ${ }^{2}$ Research Institute of Pharmaceutical Sciences, College of Pharmacy, Kyungpook National University, Daegu 702-701, \\ ${ }^{3}$ Deparment of Biochemistry, School of Medicine, Catholic University of Daegu, Daegu 705-718, \\ ${ }^{4}$ Department of Pharmacology, School of Medicine, Keimyung University, Daegu 704-701, Republic of Korea
}

\begin{abstract}
The purpose of this study is to investigate the memory enhancing effect and underlying molecular mechanism of arabinoxylan (AX), a major component of dietary fiber in wheat against scopolamine (SCO)-induced amnesia in Sprague-Dawley (SD) rats. Diverse behavior tests including Y-maze, Morris water maze, and passive avoidance tests were performed to measure cognitive functions. SCO significantly decreased the spontaneous alterations in Y-maze test and step-through latency in passive avoidance test, whereas increased time spent to find the hidden platform in Morris water maze test compared with the sham control group. In contrast, oral administration of $\mathrm{AX}(25 \mathrm{mg} / \mathrm{kg}$ and $50 \mathrm{mg} / \mathrm{kg})$ effectively reversed the SCO-induced cognitive impairments in SD rats. Furthermore, AX treatment up-regulated the expression of brain-derived neurotrophic factor (BDNF) in the cortex and hippocampus via promoting activation of cAMP response element binding protein (CREB). Therefore, our findings suggest that $A X$ can improve SCO-induced learning and memory impairment possibly through activation of CREB and up-regulation of BDNF levels, thereby exhibiting a cognition-enhancing potential.
\end{abstract}

Key Words: Arabinoxylan, Brain-derived neurotrophic factor, cAMP response element binding protein, Learning and memory, Scopolamine

\section{INTRODUCTION}

Characteristic neuropathological features of Alzheimer's disease $(A D)$, one of the most common forms of dementia, are accumulation of senile plaques, formation of neurofibrillary tangles, induction of oxidative stress and/or inflammatory responses, and disturbance in neurotransmission (Karran et al., 2011). Particularly, decreased cholinergic function is responsible for the progressive loss of memory in $A D$ and closely correlated with the severity of cognitive impairment (Craig et al., 2011). Conversely, the restoration of cholinergic function by cholinergic agonists or prolongation of the acetylcholine availability into the synaptic cleft by acetylcholinesterase $(\mathrm{AChE})$ inhibitors has been regarded as a representative therapeutic target for the treatment of AD symptoms (Brenner et al., 2008). Therefore, in the present study, we have utilized scopolamine (SCO), a muscarinic acetylcholine receptor (mA-
$\mathrm{ChR}$ ) antagonist to induce memory impairment in SpragueDawley (SD) rats as a typical screening model system for antiamnesic drugs.

Because of the limitation of current medications approved for the management of $A D$ such as relatively low efficacy, severe adverse effects for the long-term use, and ineffectiveness in the late stage of $A D$, extensive researches have been carried out to search novel and safe phytochemicals with neuroprotective properties. In this regard, an array of natural products from dietary foods with high potency and low side effects has been investigated for the prevention and/or treatment of $A D$ and one promising new candidate of interest in this study is arabinoxylan (AX).

$A X$ is the major constituent of wheat non-starch polysaccharides (NSP) accounting for approximately $2.5 \%$ of flour and $18 \%$ of bran and acts as dietary fiber in wheat (Gebruers et al., 2008; Ward et al., 2008). AX of wheat is composed of

\section{Open Access http://dx.doi.org/10.4062/biomolther.2014.063}

This is an Open Access article distributed under the terms of the Creative Commons Attribution Non-Commercial License (http://creativecommons.org/licenses/by-nc/3.0/) which permits unrestricted non-commercial use, distribution, and reproduction in any medium, provided the original work is properly cited.
Received May 29, 2014 Revised July 7, 2014 Accepted July 8, 2014 Published online Sep 30, 2014

\section{*Corresponding Author}

E-mail: pamy202@kmu.ac.kr (Jang JH), leejw@cu.ac.kr (Lee JW) Tel: +82-53-580-3866 (Jang JH), +82-53-650-4471 (Lee JW) Fax: +82-53-580-3866 (Jang JH), +82-53-621-9206 (Lee JW) 
xylose and arabinose serving as backbone and substituent, respectively. The relative ratio of arabinose to xylose $(A / X)$ is approximately 0.5 for total AX in flour (Gebruers et al., 2008; Ward et al., 2008). Previously, we have shown that hot water extract of milled wheat and total dietary fiber prepared from it improved memory impairment caused by SCO in SD rats (Jang et al., 2010). In addition, we also have reported that hot water extract of milled wheat restored memory deficit in a rat model of chronic hypoperfusion selected for mimicking vascular dementia in human (Han et al., 2010).

As an extension of the previous studies, the purpose of the present work is to investigate whether $\mathrm{AX}$, an active constituent of wheat, has a novel cognition-enhancing activity against the SCO-induced amnesia in SD rats and exhibits a preventive and/or therapeutic potential for the management of AD. In this study, basic investigations on the action of $A X$ were extended to the molecular levels by examining alterations in the phosphorylation of cAMP response element binding (CREB) protein and expression of brain-derived neurotrophic factor (BDNF).

\section{MATERIALS AND METHODS}

\section{Drugs and reagents}

Wheat AX was purchased from Megazyme (Wicklow, Ireland). SCO and anti-actin antibody were obtained from SigmaAldrich (St. Louis, MO, USA). Anti-BDNF and anti-phosphoCREB ( $p$-CREB) antibodies were the products of SantaCruz (Santa Cruz, CA, USA) and Cell Signaling (Boston, MA, USA), respectively.

\section{Experimental animals}

Male SD rats weighing 250-300 g (8-week old) were supplied from the Dae Han Bio-Link. Co., Ltd. (Eumseong-gun, Chungcheongbuk-do, South Korea) and maintained under controlled temperature $\left(21 \pm 2^{\circ} \mathrm{C}\right)$ and humidity (45-60\%) with a regular $12 \mathrm{~h}$ light $-12 \mathrm{~h}$ dark cycle (light period starting at 7 a.m.). The rats freely accessed to standard rodent chow and fresh tap water. The experimental procedure was conducted in compliance with institutional guidelines of $\mathrm{NIH}$ and Daegu Haany University for the Care and Use of Laboratory Animals.

\section{Drug administration}

The rats were divided into four groups (6-7 animals per group): sham control group, SCO model group, and SCO+ AX $(25 \mathrm{mg} / \mathrm{kg}$ and $50 \mathrm{mg} / \mathrm{kg})$ treatment groups. The rats were orally administered with normal saline or $A X, 1 \mathrm{~h}$ before starting the behavior tests. SCO $(0.75 \mathrm{mg} / \mathrm{kg}, 300 \mu \mathrm{l}$, i.p. $)$ was injected 30 min prior to the behavior tests, whereas the sham control group was injected with same volume of normal saline.

\section{Y-maze test}

Three arms of the Y-maze $(41 \mathrm{~cm}$ long, $25 \mathrm{~cm}$ high, and $10 \mathrm{~cm}$ wide) were positioned at an equal angle. Each rat was placed at the end of one arm and allowed to navigate freely for an 8-min session. In each trial, the spontaneous alternations were recorded using Ethovision System (Ethovision system, Noldus, Wageningen, Netherlands). Spontaneous alteration behavior was defined as the entry into all three arms on consecutive choices in overlapping triplet sets (e.g. ABC, BCA, $\mathrm{CAB})$. The percentage (\%) of spontaneous alternation behav- ior was calculated by dividing the total number of alternations by the total number of arm entries subtracting 2 and then multiplying by 100 : $\%$ alternation $=[($ number of alternations $) /($ total number of arm entries-2)] $\times 100$.

\section{Morris water maze test}

The Morris water maze test was conducted in accordance with the protocol previously reported (Jang et al., 2010).

\section{Step-through passive avoidance test}

The passive avoidance test apparatus (GEMINI ${ }^{\mathrm{TM}}$, San Diego, CA, USA) was consisted of two compartments $(26 \times 28 \times 16$ $\mathrm{cm}$ ), illuminated and non-illuminated chambers, equipped with an electrifiable grid floor. The two chambers were connected by an automatically operated guillotine door. During acquisition trial, rats were initially placed in the illuminated chamber and after $30 \mathrm{sec}$ of familiarization, the light was on and the door between the two compartments was opened. When the rats entered the non-illuminated chamber, the guillotine door was automatically closed and an electrical foot shock $(0.5 \mathrm{~mA})$ was delivered for $5 \mathrm{sec}$ through the stainless steel rods. The time taken to enter the non-illuminated chamber was recorded as the step-through latency. A retention trial was performed $24 \mathrm{~h}$ after training trial. The rats were placed again in the light chamber and the time to enter the dark chamber after door opening was measured without electric foot shock. If the rat did not enter the dark chamber within $300 \mathrm{sec}$, the stepthrough latency was regarded as $300 \mathrm{sec}$.

\section{Reverse transcription-polymerase chain reaction: RT-PCR}

After conducting behavior test, rats were anesthetized with pentobarbital $(50 \mathrm{mg} / \mathrm{kg}$, i.p.) and sacrified by decapitation to obtain cortical tissues. Total RNA was extracted from the rat cortex with TRI reagent (Molecular Research center, OH, USA) and then reverse transcribed for $60 \mathrm{~min}$ at $42^{\circ} \mathrm{C}$ using M-MLV reverse transcriptase (Promega, WI, USA). Amplification of cDNA was conducted by polymerase chain reaction (PCR) using synthetic primers specific to choline acetyltransferase (ChAT), $M_{1}$ type mAChR ( $\left.M_{1} m A C h R\right)$, and glyceraldehyde-3-phosphate dehydrogenase (GAPDH) as follows: ChAT, 5'-AGGGTGATCTGTTCACTCAG-3' (sence), 5'-TCTTGTTGCCTGTCATCATA-3' (antisence); M mAChR, 5'-CAGAAGTGGTGATCAAGATGCC-3' (sence), 5'-GAGCTTTTGGGAGGCTGCTT-3' (antisence); GAPDH, 5'-GCCAAGGTCATCCATGACAAC-3' (sense), 5'-AGTGTAGCCCAGGATGCCCTT-3' (antisense). The PCR products were analyzed by $1.5 \%$ agarose gel electrophoresis in Trisacetate-EDTA (TAE) buffer and examined under UV light using a gel documentation system (Geldoc 2000, Bio-Rad, Philadelphia, PA, USA) after staining with ethidium bromide (EtBr).

\section{Immunoblotting}

After completing behavior test, rats were sacrificed and the brains were dissected on an ice-cold operating table. The cortex and hippocampus were taken from the brain and homogenated thoroughly with RIPA buffer using automatic homogenizer (Precellys 24, Bertin Technologies, Montigny le Bretonneux, France). The homogenates were centrifuged at $14,000 \mathrm{~g}$ for $15 \mathrm{~min}$ on $4^{\circ} \mathrm{C}$. The concentration of isolated protein was determined by BCA protein assay kit (Pierce Biotechnology, IL, USA). Immunoblotting was performed according to the procedures previously described (Lee et al., 2011a). 


\section{Immunohistochemistry}

Rats were anesthetized with an intraperitoneal injection of pentobarbital $(50 \mathrm{mg} / \mathrm{kg})$ and perfused with phosphate buffered saline (PBS) followed by $4 \%$ cold paraformaldehyde in PBS. Brain tissues were post-fixed in the same fixative for additional $2 \mathrm{~h}$ at room temperature and then sequentially immersed in $10 \%, 20 \%$, and then $30 \%$ sucrose solution. Frozen tissue blocks were made with OTC compound (Tissue Tek, Torrance, CA, USA) and coronal brain sections (30 $\mu \mathrm{m}$ thick) were serially taken using a cryostat (Leica, Wetzlar, Germany). Immunohistochemical staining was conducted following the procedures previously reported with some modifications (Lee et al., 2011b). Correspondent slides with adhered sections were washed with $1 \%$ Triton X-100 in PBS for 10 min and then with PBS for 5 min twice. Endogenous peroxidase was inactivated by incubating the tissue sections with $1 \% \mathrm{H}_{2} \mathrm{O}_{2}$ in PBS for $30 \mathrm{~min}$. After washing with PBS for three times, the
A

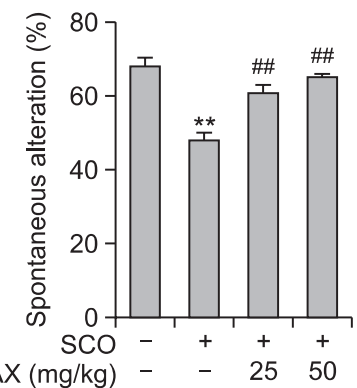

B

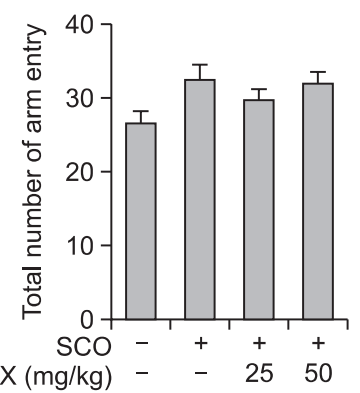

Fig. 1. Memory enhancing effect of $A X$ in $Y$-maze test. (A) Effect of $A X$ on the SCO-decreased spontaneous alternation was monitored by Y-maze test. (B) Total number of arm entries during 8-min session of $Y$-maze test. Data are presented as mean \pm S.E.M. $(n=7)$. Significant difference between groups: ${ }^{* *} p<0.01$, vehicle-treated control vs. SCO alone group; ${ }^{\#} p<0.01$, SCO alone group vs. AXtreated groups in combination with SCO. slides were blocked in $3 \%$ normal horse serum containing $0.1 \%$ Triton $\mathrm{X}-100$ for $1 \mathrm{~h}$ at room temperature and incubated with primary anti-p-CREB antibody (1:200) prepared in PBS containing $0.1 \%$ bovine serum for $24 \mathrm{~h}$ at $4^{\circ} \mathrm{C}$. After washing again three times with PBS, biotinylated secondary antibody was added for $2 \mathrm{~h}$, followed by incubation with avidin-biotin peroxidase complex (Santa Cruz) for $1 \mathrm{~h}$ at room temperature. Then slides were developed by using DAB as substrate, dehydrated with a series of ethanol solution, and mounted with $50 \%(\mathrm{v} / \mathrm{v})$ glycerol. The stained images were examined under a bright field microscope (Leica).

\section{Statistical analysis}

The data were expressed as mean values \pm standard error of the mean (S.E.M.). Statistical analysis was performed by one-way ANOVA followed by the Tukey's test for multiple comparisons using SPSS software (SPSS $12.0 \mathrm{KO}$ for windows). Differences were considered to be significant when $p$ value was less than 0.05

\section{RESULTS}

\section{Effect of AX on SCO-induced memory impairments}

Immediate working memory performance was assessed by recording spontaneous alteration behavior in Y-maze test. In this test, SCO $(0.75 \mathrm{mg} / \mathrm{kg}$, i.p. $)$ significantly decreased the percentage of spontaneous alteration (Fig. 1A). Treatment of SD rats with AX (25 or $50 \mathrm{mg} / \mathrm{kg}$ ) effectively increased \% spontaneous alteration and improved memory impairment caused by SCO in a dose-related manner (Fig. 1A). However, under the same experimental condition, the total number of arm entries was similar in each experimental group (Fig. 1B).

In another experiment, Morris water-maze was conducted to compare the spatial memory. The rats in the vehicle-treated sham control group readily learned and memorized the location of submerged hidden platform during four consecutive training days, which was reached within $30 \mathrm{sec}$ by the last day
A

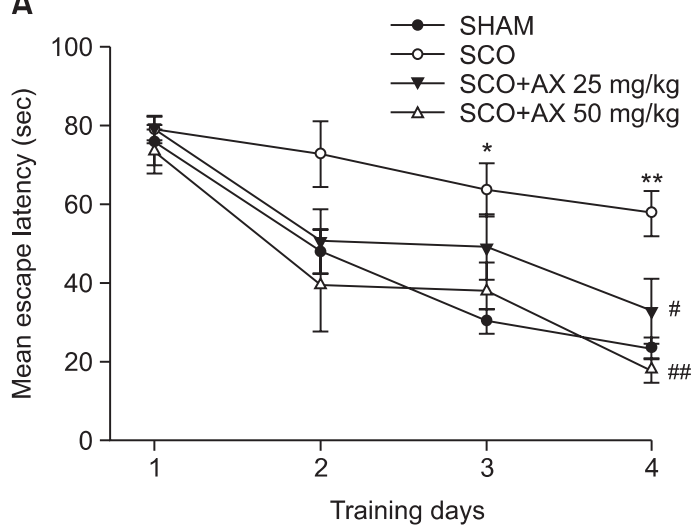

B

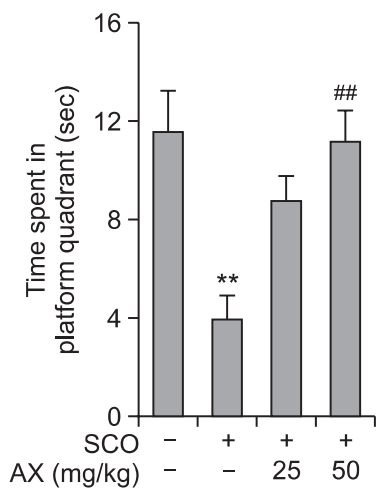

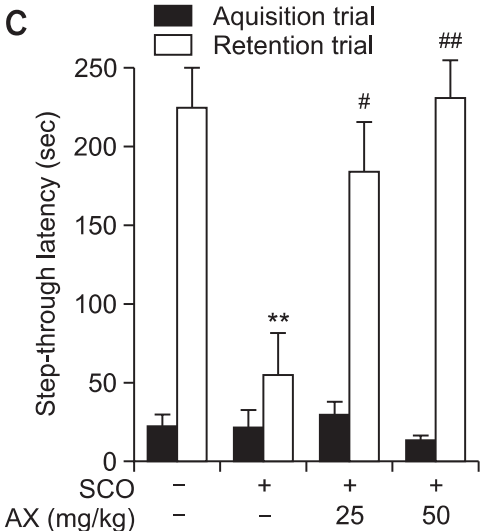

C 
A

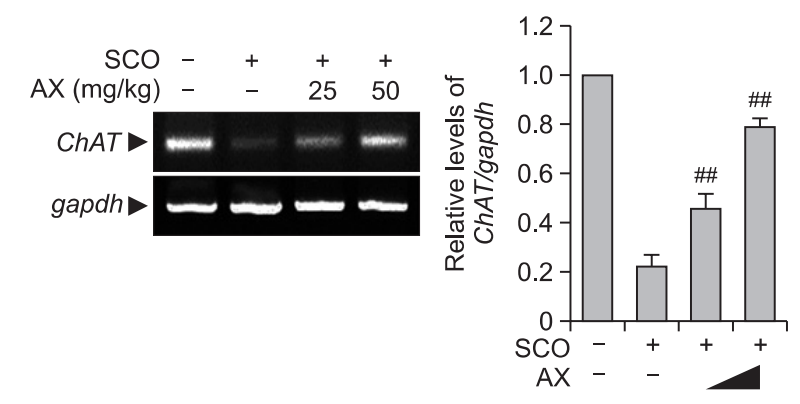

B

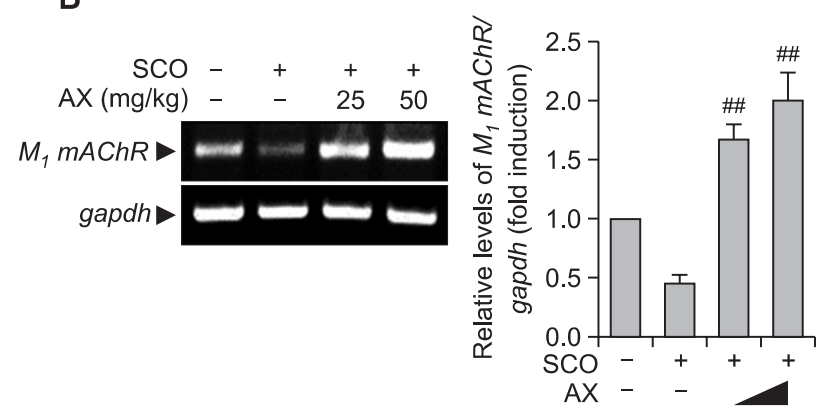

Fig. 3. Effect of $A X$ on the mRNA expression of acetylcholinerelated proteins The mRNA expression of enzymes involved in the synthesis of acetylcholine (A) and $M_{1}$ type mAChR (B) was analyzed by RT-PCR ( $\mathrm{n}=3$ per lane). mRNA levels of gapdh were determined to confirm equal amount of mRNA loading. Quantitative values for the relative mRNA expression were calculated from three independent experiments and represented in the right panels $(n=9)$. Significant difference between groups : ${ }^{\#} p<0.01$, SCO alone group vs. AX-treated groups in combination with SCO.

of the training period (Fig. 2A). Conversely, the performance in the SCO alone-injected group was significantly impaired. However, repeated daily administration of AX ( 25 or $50 \mathrm{mg} / \mathrm{kg}$ ) reduced mean escape latencies starting from the second day and particularly $50 \mathrm{mg} / \mathrm{kg}$ of AX-administered group exhibited almost similar mean escape latency to the vehicle-treated sham control group during four consecutive training days (Fig. 2A). Immediately after the last training session on day 4 , the platform was removed and probe test was conducted for $30 \mathrm{~s}$. The time spent in the target quadrant to search for the removed platform was monitored. The sham control and AXtreated groups spent more time in the probe quadrant compared with SCO alone-treated group (Fig. 2B) suggesting the retention of learning and memory by oral administration of $A X$.

To confirm the effect of $A X$ on the SCO-induced learning and memory impairments, passive avoidance test was performed. In the acquisition trial, there was no significant difference in step-through latency time among experimental groups (Fig. 2C). However, during retention trial, the step-through latency was dramatically decreased in the SCO-injected rats and SCO-reduced step-through latency was effectively restored by oral administration of increasing doses of AX (25 and $50 \mathrm{mg} / \mathrm{kg}$ ) (Fig. 2C).

\section{Effect of AX on the expression of enzymes and receptors related with acetylcholine}

To verify the effect of $A X$ on the acetylcholine metabolism, the mRNA levels of enzymes and receptors involved in the synthesis as well as pharmacological action of acetylcholine were monitored by RT-PCR. In the cortex, SCO decreased the mRNA expression of ChAT which plays a key role in the formation of acetylcholine (Fig. 3A). SCO-induced a decrease in the ChAT mRNA levels was restored by AX ( 25 or $50 \mathrm{mg} / \mathrm{kg}$ ) in a dose-dependent manner (Fig. 3A). However, under the same experimental condition the mRNA expression of AChE, acetylcholine decomposing enzyme, was not much affected by SCO or AX treatment (data not shown). In another experiment, SCO injection also suppressed mRNA expression of $M$ type of muscarinic acetylcholine receptor $\left(M_{1} m A C h R\right)$, which was significantly up-regulated by oral administration of $A X(25$ or $50 \mathrm{mg} / \mathrm{kg}$ ) dose-dependently (Fig. 3B).

\section{Effect of AX on the expression of BDNF and activation of CREB}

To further elucidate the molecular mechanisms underlying the memory enhancing effect of $A X$, we have examined the expression of BDNF in the cortical and hippocampal brain tissues. Treatment of SD rats with AX (25 or $50 \mathrm{mg} / \mathrm{kg}$ ) dosedependently increased the protein levels of BDNF which was decreased by SCO in the cortex and hippocampus as well (Fig. 4A). To further determine an upstream regulator for the up-regulation of BDNF by $A X$, the possible involvement of CREB was investigated. Proteins from cortical or hippocampal tissue lysates were analyzed by Western blot analysis with p-CREB specific antibody. SCO-decreased phosphorylation of CREB was marked restored by oral administration of $A X$ with maximal induction at the dose of $50 \mathrm{mg} / \mathrm{kg}$ (Fig. 4B). We also verified activation of $p$-CREB by $A X(50 \mathrm{mg} / \mathrm{kg})$ in the cortex as well as hippocampus (Fig. 4C) using immunohistochemistry. As a result, the phosphorylation of CREB was evident after $A X$ treatment in accordance with the Western blot data of BDNF.

\section{DISCUSSION}

In this study, we have investigated the neuroprotective effects of AX against SCO-induced cognitive dysfunction in SD rats. First, to induce learning and memory impairment, we have intraperitonically injected SCO in SD rats and conducted a series of behavior tests. In the Y-maze test, SCO-treated rats showed decreased spontaneous alterations compared with that of vehicle-treated animals, which was improved by AX pretreatment. In Morris water-maze task, SCO-treated rats took longer time to find platform than the rats in sham control group and AX-treated group in combination with SCO easily found the location of hidden platform. In the passive avoidance test, SCO treatment reduced step-through latency, which was effectively restored by oral daily administration of $A X$.

As a molecular mechanism underlying the memory enhancing effect of $A X$, we showed that $A X$ treatment increased phosphorylation of CREB, an activated form of CREB, and stimulated the expression of BDNF, ChAT and $M_{1} m A C h R$ genes. It has been reported that BDNF, ChAT and $\mathrm{MAChR}$ genes have CRE, the binding site of CREB (Zhang et al., 2005) implying that phosphorylation of CREB can stimulate the transcription of aforementioned genes. Indeed, phosphorylation of CREB ameliorated stresses generated by diverse pathophysiological conditions such as AD (Jin et al., 2009), ischemia (Kim et al., 2009), aging (Asanuma et al., 1996; Hu et al., 2010) in as- 
A

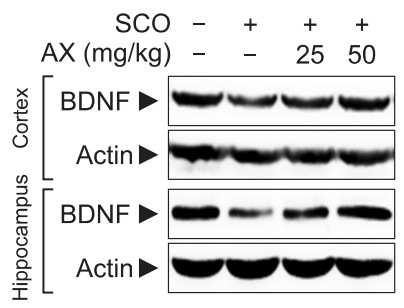

B

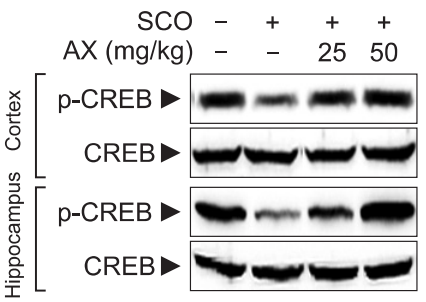

Cortex

Hippocampus

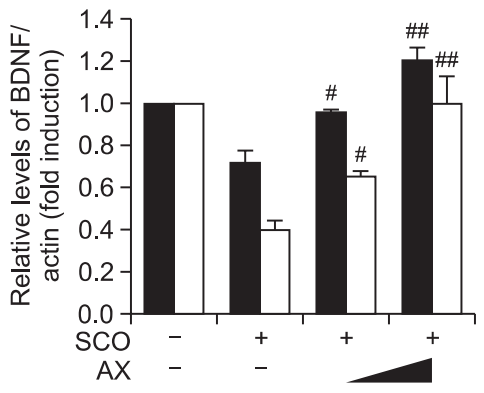

Cortex

Hippocampus

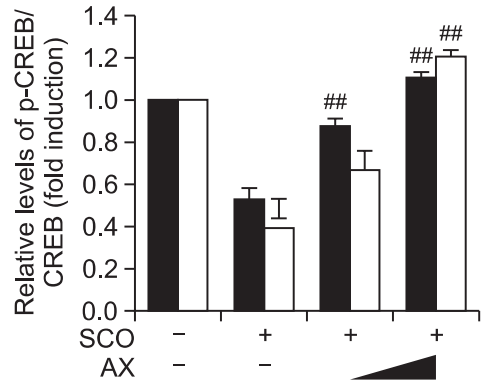

C

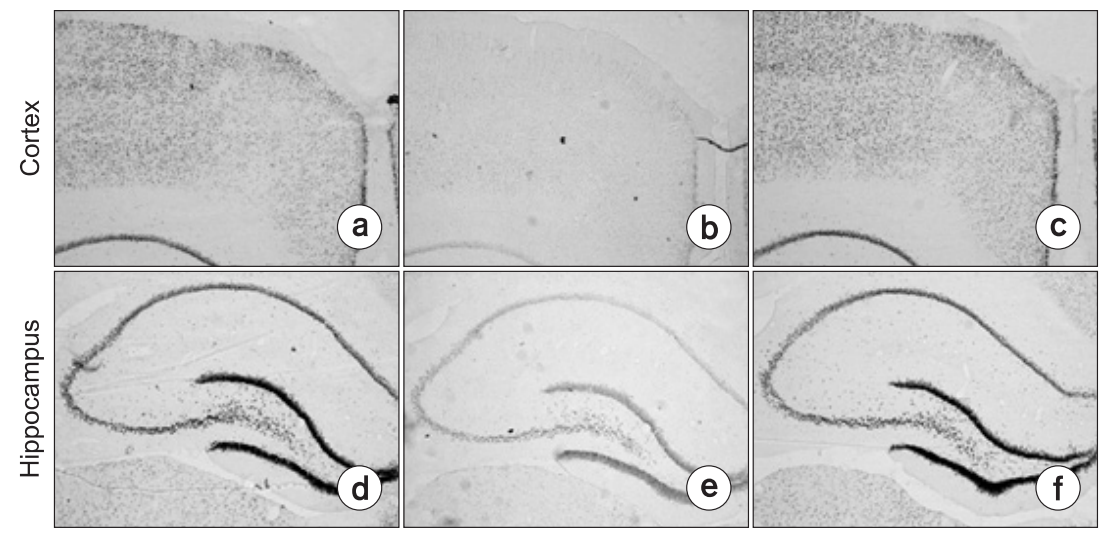

Fig. 4. Effect of $A X$ on the protein levels of BDNF and activation of CREB The protein expression of BDNF (A) and p-CREB (B) was examined by Western blot analysis ( $n=3$ per lane). Proteins from tissue lysates were analyzed by using BDNF or $p-C R E B$ specific antibody. Actin expression levels were monitored to ensure equal amount of protein loading. Relative protein expression calculated from three independent experiments was represented in the right panel $(n=9)$. Significant difference between groups : ${ }^{\#}<<0.05$ and ${ }^{\# \#} p<0.01$, SCO alone group vs. AX-treated groups in combination with SCO. (C) Activation of CREB was verified by immunohistochemistry utilizing anti-p-CREB antibody. a and $\mathrm{d}$ : sham control, b and e : SCO alone $(0.75 \mathrm{mg} / \mathrm{kg})$ group, c and $\mathrm{f}: \mathrm{SCO}(0.75 \mathrm{mg} / \mathrm{kg})+\mathrm{AX}(50 \mathrm{mg} / \mathrm{kg})$.

sociation with BDNF (Jin et al., 2009; Kim et al., 2009), ChAT (Asanuma et al., 1996; Jin et al., 2009), and mAChR (Jin et al., 2009; Hu et al., 2010). Particularly, the activation of CREB and increased expression of BDNF are involved in enhancement of long-term memory (Cunha et al., 2010; Bitner, 2012). In addition, up-regulated expression of ChAT and $\mathrm{mAChR}$ results in an increased production of acetylcholine and amplification of signal transduction with acetylcholine acting as a ligand. Thus, CREB-mediated expression of BDNF, ChAT and mAChR by $A X$ may contribute to the improvement of memory impaired by SCO.

In accordance with our findings, a wide range of phytochemicals have been reported to have neuroprotective and memory enhancing effects via CREB-BDNF signals. Triterpenoid saponin PGS32 (polygalasaponin XXXII) isolated from the roots of Polygala tenuifolia Willd. prevented SCO-induced cognitive impairments possibly through improvement of synaptic transmission, activation of the mitogen-activated protein kinase (MAPK) and CREB, and enhancement of BDNF levels (Xue et al., 2009). Kalopanaxsaponins A and B derived 
from stem-bark of Kalopanax pictus ameliorated SCO-induced memory deficits by inducing BDNF and p-CREB expression and conversely inhibiting AChE activity as well (Joh et al., 2011).

To determine which compounds might be responsible for improving memory in rats administered with SCO, we have to follow the passages that the AX undergoes in the gastrointestinal tract. Zhang et al. (Zhang et al., 2003) demonstrated that approximately $3 \%$ of arabinose on $\mathrm{AX}$ was released in 3 $\mathrm{h}$ of incubation at $\mathrm{pH} 3$. Subsequently the remaining $\mathrm{AX}$ entering the small intestine is suitable for the breakdown as it is not a substrate for enzymatic digestion in the small intestine. Finally, the remaining $A X$ entering the large intestine is first degraded into monosaccharides such as arabinose and xylose by bacteria inhabiting in the large intestine, followed by fermentation into short chain fatty acids (SCFA) such as butyrate, propionate and acetate (Broekaert et al., 2011). As a result, both the monosaccharides generated in the small and large intestines and SCFA formed in the large intestine are promising candidates that can be absorbed into the blood and reach the brain. Arabinose (Eidelberg et al., 1967) and butyrate (Dienel et al., 2001) have been reported to penetrate across blood-brain barrier (BBB) and be transported into the brain. However, mechanisms by which arabinose and/or $x y-$ lose might play in enhancing learning and memory functions have not been elucidated yet, although oral administration of arabinose attenuated brain injury by inhibiting inflammatory responses caused by hypoperfusion in SD rats (Han et al., 2010). On the other hand, memory enhancing mechanisms of butyrate are beginning to be elucidated. Butyrate can be metabolized to produce ATP that can be converted to CAMP by adenylyl cyclase. Therefore, consequent increase of cAMP activates protein kinase $A$ (PKA) leading to phosphorylation of CREB (Wang et al., 2012). Thus, contribution of AX to enhancing memory can be explained, at least in part, by conversion of AX into arabinose and butyrate.

In summary, we demonstrated that $A X$ improved learning and memory functions in SD rats with SCO-induced amnesia. This cognition enhancing effect of $A X$ was mediated partly through activation of CREB cascade and subsequent expression of BDNF. To translate the novel effect of AX in CNS into clinical applications for the improvement of cognitive functions, the pharmacology and mode of action of its active components should be further investigated.

\section{ACKNOWLEDGMENTS}

This research was supported by Bio-industry Technology Development Program, Ministry for Food, Agriculture, Forestry and Fisheries, Republic of Korea.

\section{REFERENCES}

Asanuma, M., Nishibayashi, S., Iwata, E., Kondo, Y., Nakanishi, T., Vargas, M. G. and Ogawa, N. (1996) Alterations of cAMP response element-binding activity in the aged rat brain in response to administration of rolipram, a cAMP-specific phosphodiesterase inhibitor. Brain Res. Mol. Brain Res. 41, 210-215.

Bitner, R. C. (2012) Cyclic AMP response element-binding protein (CREB) phosphorylation: A mechanistic marker in the development of memory enhancing Alzheimer's disease therapeutics. Biochem. Pharmacol. 83, 705-714.

Brenner, T., Nizri, E., Irony-Tur-Sinai, M., Hamra-Amitay, Y. and Wirguin, I. (2008) Acetylcholinesterase inhibitors and cholinergic modulation in myasthenia gravis and neuroinflammation. J. Neuroimmunol. 201-202, 121-127.

Broekaert, W. F., Courtin, C. M., Verbeke, K., Wiele, T. V. D., Verstraete, W. and Delcour, J. A. (2011) Prebiotic and other health-related effects of cereal-derived arabinoxylans, arabinoxylan-oligosaccharides, and xylooligosaccharides. Crit. Rev. Food Sci. Nutr. 51, 178-194.

Craig, L. A., Hong, N. S. and McDonald, R. J. (2011) Revisiting the cholinergic hypothesis in the development of Alzheimer's disease. Neurosci. Biobehav. Rev. 35, 1397-1409.

Cunha, C., Brambilla, R. and Thomas, K. L. (2010) A simple role for BDNF in learning and memory? Front. Mol. Neurosci. 3, 1.

Dienel, G. A., Liu, K. and Cruz, N. F. (2001) Local uptake of ${ }^{14} \mathrm{C}$-labeled acetate and butyrate in rat brain in vivo during spreading cortical depression. J. Neurosci. Res. 66, 812-820.

Eidelberg, E., Fishman, J. and Hams, M. L. (1967) Penetration of sugars across the blood-brain barrier. J. Physiol. 191, 47-57.

Gebruers, K., Dornez, E., Boros, D., Fraś, A., Dynkowska, W., Bedo, Z., Rakszegi, M., Delcour, J. A. and Courtin, C. M. (2008) Variation in the content of dietary fiber and components thereof in wheats in the HEALTHGRAIN diversity screen. J. Agric. Food Chem. 56, 9740-9749.

Han, H. S., Jang, J. H., Jang, J. H., Choi, J. S., Kim, Y. J., Lee, C., Lim, S. H., Lee, H. K. and Lee, J. (2010) Water extract of Triticum aestivum $\mathrm{L}$. and its components demonstrate protective effect in a model of vascular dementia. J. Med. Food 13, 572-578.

Hu, H., Zhang, R., Zhang, Y., Xia, Z. and Hu, Y. (2010) Role of CREB in the regulatory action of sarsasapogenin on muscarinic $\mathrm{M}_{1}$ receptor density during cell aging. FEBS Lett. 584, 1549-1552.

Jang, J. H., Kim, C. Y., Lim, S. H., Yang, C. H., Song, K. S., Han, H. S., Lee, H. K. and Lee, J. (2010) Neuroprotective effects of Triticum aestivum L. against beta-amyloid cell death and memory impairments. Phytother. Res. 24, 76-84.

Jin, C. H., Shin, E. J., Park, J. B., Jang, C. G., Li, Z., Kim, M. S., Koo K. H., Yoon, H. J., Park, S. J., Choi, W. C., Yamada, K., Nabeshima, T. and Kim, H. C. (2009) Fustin flavonoid attenuates $\beta$-amyloid (1-42)-induced learning impairment. J. Neurosci. Res. 87, 36583670 .

Joh, E. H., Lee, I. A. and Kim, D. H. (2011) Kalopanaxsaponins A and B isolated from Kalopanax pictus ameliorate memory deficits in mice. Phytother. Res. 26, 546-551.

Karran, E., Mercken, M. and De Strooper, B. (2011) The amyloid cascade hypothesis for Alzheimer's disease: an appraisal for the development of therapeutics. Nat. Rev. Drug Discov. 10, 698-712.

Kim, H. J., Leeds, P. and Chuang, D. M. (2009) The HDAC inhibitor, sodium butyrate, stimulates neurogenesis in the ischemic brain. $J$. Neurochem. 110, 1226-1240.

Lee, C., Park, G. H., Kim, C. Y. and Jang, J. H. (2011a) [6]-Gingerol attenuates $\beta$-amyloid-induced oxidative cell death via fortifying cellular antioxidant defense system. Food Chem. Toxicol. 49, 1261 1269.

Lee, C., Park, G. H. and Jang, J. H. (2011b) Cellular antioxidant adaptive survival response to 6-hydroxydopamine-induced nitrosative cell death in C6 glioma cells. Toxicology 283, 118-128.

Ward, J. L., Poutanen, K., Gebruers, K., Piironen, V., Lampi, A. M., Nyström, L., Andersson, A. A., Aman, P., Boros, D., Rakszegi, M. Bedo, Z. and Shewry, P. R. (2008) The HEALTHGRAIN cereal diversity screen: concept, results, and prospects. J. Agric. Food Chem. 56, 9699-9709.

Wang, A., Si, H., Liu, D. and Jiang, H. (2012) Butyrate activates the cAMP-protein kinase A-cAMP response element-binding protein signaling pathway in Caco-2 cells. J. Nutr. 142, 1-6.

Xue, W., Hu, J. F., Yuan, Y. H., Sun, J. D., Li, B. Y., Zhang, D. M., Li, C. J. and Chen, N. H. (2009) Polygalasaponin XXXII from Polygala tenuifolia root improves hippocampal-dependent learning and memory. Acta Pharmacol. Sin. 30, 1211-1219.

Zhang, P., Zhang, Q. and Whistler, R. L. (2003) L-Arabinose release from arabinoxylan and arabinogalactan under potential gastric 
Kim et al. Memory Enhancing Effect of Arabinoxylan

aciditities. Cereal Chem. 80, 252-254.

Zhang, X., Odom, D. T., Koo, S. H., Conkright, M. D., Canettieri, G., Best, J., Chen, H., Jenner, R., Herbolsheimer, E., Jacobsen, E., Kadam, S., Ecker, J. R., Emerson, B., Hogenesch, J. B., Unterman,
T., Young, R. A. and Montminy, M. (2005) Genome-wide analysis of cAMP-response element binding protein occupancy, phosphorylation, and target gene activation in human tissues. Proc. Natl. Acad. Sci. U.S.A. 102, 4459-4464. 